\title{
Centro de trasplante hepático en México con bajo volumen y excelentes resultados
}

\author{
Mario Vilatobá1, Miguel Ángel Mercado², Alan Gabriel Contreras-Saldivar', \\ Rafael Paulino Leal-Villalpando ${ }^{3}$, Jorge Zamudio-Bautista ${ }^{3}$, Ignacio García-Juárez ${ }^{4}$ \\ y Armando Gamboa-Domínguez ${ }^{5}$ \\ ${ }^{1}$ Departamento de Trasplantes; ${ }^{2}$ Dirección de Cirugía; ${ }^{3}$ Departamento de Anestesiología; ${ }^{4}$ Departamento de Gastroenterología; ${ }^{5}$ Departamento de \\ Patología. Instituto Nacional de Ciencias Médicas y Nutrición Salvador Zubirán, Ciudad de México, México
}

\section{Resumen}

Antecedentes: El trasplante hepático ortotópico (THO) es el tratamiento de elección para la insuficiencia hepática terminal. Numerosos estudios muestran una relación inversa entre el número de procedimientos y la mortalidad operatoria. Objetivo: El objetivo de este estudio es mostrar los resultados de nuestro centro y determinar si puede tener resultados equiparables a los obtenidos en centros de alto volumen. Método: Es un estudio retrospectivo en el que se analizó la información de pacientes con THO en nuestra institución, de 1985 al 31 de diciembre de 2012. Dependiendo de la fecha del THO, el estudio se dividió en tres etapas: etapa 1, de 1985 a 1999; etapa 2, de 2000 a 2007; y etapa 3, de 2008 a 2012. En las etapas 1, 2 y 3 se realizaron 22, 37 y 56 THO, respectivamente. Resultados: La mortalidad perioperatoria fue menor de manera significativa en la etapa 3 en comparación con las etapas 1 y 2 (3.5 vs. 50 y 21.7\%; $p=0.001$ ). La supervivencia de los pacientes a 1 y 5 años fue mejor en la etapa 3 (94.4 y 87.8\%) que en la etapa 2 (77.6 y 66.17\%) y en la etapa 1 (47 y 29\%) (p=0.001). Conclusión: En conclusión, los resultados actuales en THO en nuestro programa son excelentes, a pesar de ser un centro de bajo volumen.

PALABRAS CLAVE: Trasplante. Hígado. Resultados. Bajo volumen.

\begin{abstract}
Background: Orthotopic liver transplantation (OLT) is the treatment of choice for end stage liver disease. Many studies show an inverse relationship between the number of procedures and operative mortality. Objective: The objective of the study is to show the results of our center and determine if it can have comparable results to high volumen centers. Method: This is a retrospective study which analyzed the information of patients with OLT at our institution from 1985 to December 31, 2012. Depending on date of transplantation, the study was divided into three stages. Stage 1: from 1985 to 1999. Stage 2: from 2000 to 2007. Stage 3: from 2008 to 2012. In the 1, 2 and 3 stage 22, 37 and 56 OLT were performed respectively. Results: Perioperative mortality was significantly lower between Stage 3 vs. Stage 1 and $2(3.5 \%$ vs. $50 \%$ and $21.7 \%, p=0.001)$. Patient survival was also better at 1 and 5 years at Stage $3(94.4 \%, 87.8 \%)$ vs. era 2 (77.6\%, 66.17\%) and Stage 1 (47\% and $29 \%)(p=0.001)$. Conclusion: In conclusion, the present results of OLT at our program are excellent despite being a low-volume center.
\end{abstract}

KEY WORDS: Transplant. Liver. Results. Low-volume.

\author{
Correspondencia: \\ Mario Vilatobá \\ Avda. Vasco de Quiroga, 15 \\ Col. Belisario Domínguez \\ Sección XVI, Del. Tlalpan \\ C.P. 14080 , Ciudad de México, México \\ E-mail: mvilatoba@hotmail.com
}

Fecha de recepción en versión modificada: 06-09-2016

Fecha de aceptación: 08-09-2016

DOI:10.24875/GMM.17002673
Gac Med Mex. 2017;153:441-449

Contents available at PubMed www.gacetamedicademexico.com 


\section{Introducción}

El trasplante hepático ortotópico (THO) es el tratamiento de elección para la insuficiencia hepática terminal, aguda o crónica, y para algún tipo de tumores ${ }^{1}$. A pesar de todos los avances en cirugía y cuidados perioperatorios, el THO continúa siendo un procedimiento complejo, con un alto índice de complicaciones y una mortalidad no despreciable. Numerosos estudios han documentado una relación inversa entre el número de procedimientos quirúrgicos complejos y la mortalidad postoperatoria, definida como muerte en los siguientes 30 o 90 días tras un procedimiento quirúrgico ${ }^{2,3}$. Inclusive, estudios realizados más de una década después muestran esta misma relación ${ }^{4}$. En 1994, Hosenpud, et al. ${ }^{5}$ analizaron la supervivencia dependiendo del volumen de trasplantes cardiacos y encontraron que el riesgo de muerte era un $31.1 \%$ mayor a 12 meses postrasplante en un centro que realizaba menos de nueve trasplantes por año, en comparación con aquel que realizaba más de nueve. En el caso específico del THO, en 1999 Edwards, et al. ${ }^{6}$ reportaron que la mortalidad era menor en los centros con más de 20 trasplantes por año, en comparación con centros que realizaban menos de 20 . Sin embargo, en publicaciones más recientes, posteriores a la utilización del MELD (siglas en inglés de Model for End-Stage Liver Disease), pareciera que los resultados no se ven afectados si se hacen más o menos de $20 \mathrm{THO}$ por año $0^{7-11}$. Por otro lado, nunca se ha hablado de la diferencia de un centro de bajo volumen y un centro de muy bajo volumen. Es decir, nunca se ha marcado un número mínimo como prohibitivo para realizar este tipo de procedimientos tan complejos.

En México, a pesar de que la cirrosis hepática es la segunda causa de muerte en la población económicamente activa ${ }^{12}$, el número de THO que se realizan por millón de habitantes es de los más bajos de Latinoamérica. A partir del año 2003 se ha mantenido en una meseta de 100-150 trasplantes por año en todo el país. Por lo tanto, el número de trasplantes que se realiza por centro es en general menor de 10 , lo que dificulta que algún centro que realiza este procedimiento se convierta en un centro de alto volumen ${ }^{13}$.

El siguiente estudio tiene por objetivo mostrar los resultados del THO en nuestro Instituto desde sus inicios, y tratar de determinar si un centro de bajo volumen, como el nuestro, puede tener adecuados resultados, equiparables a los centros de alto volumen de los EE.UU. y Europa.

\section{Método}

Se trata de un estudio retrospectivo en el que se analizó la información de pacientes que recibieron un THO de donante fallecido en el Instituto Nacional de Ciencias Médicas y Nutrición Salvador Zubirán (INCMNSZ) en el periodo de 1985 hasta el 31 de diciembre de 2012, excepto aquellos que recibieron un trasplante simultáneo de hígado y riñón o un trasplante de hígado dividido. La recolección de datos se llevó a cabo a partir de los registros clínicos electrónicos e impresos. A partir del año 2008, la recolección de datos se realizó de manera prolectiva. Las variables clínicas y demográficas fueron registradas tanto para describir la población como para evaluar la comparabilidad de los grupos por etapas. Como desenlaces principales se consideraron la mortalidad y la morbilidad perioperatoria, y la supervivencia a 1, 3 y 5 años. Analizamos variables determinantes en el transoperatorio, como sangrado, tiempo quirúrgico, tiempo de isquemia fría (tiempo desde que se extrajo el injerto y se almacenó en la hielera hasta que se sacó para su implante), tiempo de isquemia tibia (tiempo desde que el órgano es sacado de la solución de preservación para implantarlo hasta que es reperfundido), requerimiento de hemoderivados y evolución posquirúrgica como días de estancia en la unidad de terapia intensiva (UTI), días de estancia intrahospitalaria y complicaciones posquirúrgicas.

Solo se cuenta con la información demográfica y clínica de los donantes a partir del año 2008. El análisis estadístico se realizó con ayuda del paquete STATA 10.0.

El primer análisis descriptivo reportado es global, con fines de mostrar la experiencia en el INCMNSZ. El segundo análisis se realizó para fines de determinar la evolución del programa conforme al número de procedimientos realizados, tomando como puntos de corte temporales los descritos en el siguiente párrafo.

Con fines comparativos, los receptores del estudio se dividieron en tres etapas históricas dependiendo de la fecha en que recibieron el trasplante: etapa 1, de 1985 a 1999; etapa 2, de 2000 a 2007; y etapa 3, de 2008 a 2012.

En el análisis univariado, los datos cuantitativos se reportaron como media o mediana con sus respectivas dispersiones dependiendo de la distribución, y las 
variables categóricas se reportaron como frecuencias absolutas y relativas.

La comparación de las mediciones en las variables clínicas y demográficas pretrasplante para verificar la homogeneidad poblacional de los grupos se realizó con análisis de la varianza (ANOVA).

El análisis bivariado de los desenlaces se hizo con pruebas de hipótesis para contraste de medias, en los casos en que se observó diferencia significativa en el ANOVA. La supervivencia de las muestras fue estimada con el método de Kaplan-Meier. Determinamos significancia estadística un valor de $p<0.05$.

\section{Resultados}

De 1985 a 2012 se realizaron 117 THO. Como situaciones especiales, se realizaron un trasplante de hígado-riñón en un paciente con enfermedad renal en etapa terminal y un trasplante de hígado dividido, por lo que estos casos no fueron considerados en el análisis. Al final se recolectaron datos de 115 THO de donantes fallecidos realizados en 112 pacientes (tres retrasplantes).

En las etapas 1, 2 y 3 se realizaron 22, 37 y 56 trasplantes, respectivamente. En la tabla 1 se presentan las variables clínicas y demográficas divididas por etapas, que incluyen el tiempo en lista de espera y la puntuación de MELD. Podemos observar cómo el tiempo en lista de espera disminuyó de manera drástica en la última etapa (etapa 1: 365 días; etapa 2: 675 días; etapa 3: 80 días) gracias al incremento de donadores y a la instauración del MELD para asignar los órganos en el año 2008. También podemos observar cómo se incrementó el MELD entre las etapas 2 y 3 (14.5 vs. 18.8), lo que indica que se operaron pacientes más graves en la última etapa, aunque esto no mostro diferencia significativa $(p=0.42)$. Las principales etiologías, de manera global, fueron el virus de la hepatitis $\mathrm{C}$, enfermedades autoinmunes (cirrosis biliar primaria, colangitis esclerosante primaria y autoinmune), y consumo de alcohol y esteatosis hepática; no existió diferencia significativa en la etiología en las diferentes etapas (Tabla 2).

En la tabla 3 se resumen las variables asociadas al perioperatorio, como el tipo de cirugía, los tiempos de isquemia fría y tibia, la utilización de productos sanguíneos, los tiempos de estancia en la UTI y de hospitalización, y mortalidad perioperatoria, entre otras. En las etapas 1 y 2, desafortunadamente, no se cuenta con el tiempo de isquemia fría y tibia. Tanto el tiempo quirúrgico como la utilización de productos sanguíneos y el sangrado fueron menores, de manera estadísticamente significativa, en la etapa 2 comparada con las etapas 1 y 3 . Sin embargo, las reoperaciones, el tiempo en la UTI y la mortalidad perioperatoria fueron menores de manera significativa en la etapa 3 en comparación con las etapas 1 y 2 .

En las tablas 4 a 6 se resumen las complicaciones médicas (tempranas y tardías) y quirúrgicas que se presentaron en el periodo perioperatorio por etapas. Se observa un mayor porcentaje de complicaciones médicas en la etapa 3 que en la 2 (48\% vs. 43\%), pero no así en lo que respecta a las complicaciones quirúrgicas, que fueron mayores en las etapas 1 y 2 que en la etapa $3(36.3,48.6$ y $21.4 \%)$.

En cuanto a la gravedad de las complicaciones con la clasificación de Clavien, observamos un mayor número de complicaciones I y II en la etapa 3; sin embargo, las complicaciones graves (III-V) fueron más comunes en las etapas 1 y 2 (Tabla 7).

En la figura 1 se presenta la evolución del uso de inmunosupresores con el paso del tiempo, y se observa que en un inicio el esquema de inmunosupresión más común era con ciclosporina y azatioprina, y en la

Tabla 1. Variables demográficas y MELD divididos por etapas

\begin{tabular}{|c|c|c|c|c|}
\hline Variable & $\begin{array}{c}\text { Etapa } 1 \\
(1985-1999) \\
n=22\end{array}$ & $\begin{array}{c}\text { Etapa 2 } \\
(2000-2007) \\
n=37\end{array}$ & $\begin{array}{c}\text { Etapa } 3 \\
(2008-2012) \\
n=56\end{array}$ & $\mathrm{p}$ \\
\hline Días en lista de espera* & $365(24-365)$ & $675(325-1180)$ & $80(47-302)$ & 0.001 \\
\hline $\mathrm{Edad}^{+}$ & $36.3 \pm 13.3$ & $45.27 \pm 11.69$ & $45.46 \pm 12.08$ & 0.01 \\
\hline Sexo (\% hombres) & 38 & 51 & 51 & 0.54 \\
\hline $\mathrm{IMC}^{+}$ & $23.8 \pm 4.83$ & $25.35 \pm 4.14$ & $24.47 \pm 3.75$ & 0.40 \\
\hline MELD* & $18.4(6-40)$ & $14.5(6-38)$ & $18.8(7-40)$ & 0.42 \\
\hline
\end{tabular}

*Mediana (rango intercuartílico).

†Media desviación estándar.

IMC: índice de masa corporal; MELD: Model for End-Stage Liver Disease. 
actualidad el más utilizado es con anticuerpos monoclonales, micofenolato de mofetilo y tacrolimus. En la figura 2 se muestra la supervivencia de los receptores a 1, 3 y 5 años dividida por etapas, y podemos apreciar una mejor supervivencia conforme avanzan las etapas, de manera estadísticamente significativa.

\section{Donantes}

Desafortunadamente, en las etapas 1 y 2 no contamos con datos de los donadores; sin embargo, por estipulación del protocolo de THO del INCMNSZ, en la etapa 2 solo se consideraron donadores ideales por el índice de riesgo del donador ${ }^{14}$. Es importante mencionar que todos los trasplantes realizados fueron de donador fallecido diagnosticado con muerte encefálica con corazón latiendo. En la tabla 8 se resumen las características clínicas y demográficas de los donadores de la etapa 3. Los fines de esta descripción son poblacionales, por lo que no se realizó ningún análisis de estos datos con la evolución y el desenlace de los receptores.

Tabla 2. Etiología de los pacientes que recibieron THO dividida por etapas

\begin{tabular}{lcccc}
\hline & $\begin{array}{c}\text { Etapa } \mathbf{1} \\
\mathbf{n = 2 2} \\
\mathbf{n}(\%)\end{array}$ & $\begin{array}{c}\text { Etapa } \\
\mathbf{n = 3 7} \\
\mathbf{n}(\%)\end{array}$ & $\begin{array}{c}\text { Etapa } \mathbf{3} \\
\mathbf{n}=\mathbf{5 6} \\
\mathbf{n}(\%)\end{array}$ & $\mathbf{p}$ \\
\hline VHC & $5(22.72)$ & $11(29.72)$ & $23(41.07)$ & 0.247 \\
Autoinmune & $7(31.81)$ & $14(37.83)$ & $13(23.21)$ & 0.308 \\
Alcohol & $5(22.72)$ & $2(5.4)$ & $4(7.14)$ & 0.063 \\
NASH & 0 & 0 & $5(8.92)$ & $\mathrm{NA}$ \\
Otras & $5(22.72)$ & $10(27.02)$ & $11(19.64)$ & 0.707 \\
\hline
\end{tabular}

Autoinmune: colangitis biliar primaria, colangitis esclerosante primaria, hepatitis autoinmune; NA: no aplica; NASH: esteatohepatitis no alcohólica; VHC: virus de la hepatitis C.

Tabla 3. Variables perioperatorias divididas por etapas

\begin{tabular}{|c|c|c|c|c|}
\hline Variable & $\begin{array}{c}\text { Etapa } 1 \\
n=22\end{array}$ & $\begin{array}{c}\text { Etapa } 2 \\
\mathrm{n}=37\end{array}$ & $\begin{array}{c}\text { Etapa } 3 \\
n=56\end{array}$ & $p$ \\
\hline \multicolumn{5}{|l|}{ Tipo de trasplante } \\
\hline Bypass & 17 & - & - & \\
\hline Piggyback & - & 2 & 1 & \\
\hline Exclusión total & 4 & 35 & 55 & \\
\hline Aux. heterotópico & 1 & - & - & \\
\hline TIF h (RI) & SD & SD & $9(5-12)$ & NA \\
\hline TIT min (RI) & SD & SD & $55(30-75)$ & NA \\
\hline $\mathrm{TQ} \times \mathrm{h}(\mathrm{RI})$ & $10.6(6-17)$ & $6.5(4.5-9)$ & $7.2(4-13)$ & $<0.001$ \\
\hline$P G n .^{\circ}(R I)$ & $16.8(4-57)$ & $9.5(3-48)$ & $11(2-33)$ & 0.017 \\
\hline PFC n. ${ }^{\circ}(\mathrm{RI})$ & $13.3(2-34)$ & $4.6(2-18)$ & $9.9(2-28)$ & 0.001 \\
\hline Sangrado litros (RI) & $5.7(0.9-20)$ & $3.9(1-12)$ & $6.4(1-24)$ & 0.058 \\
\hline Días UTI (RI) & $8.4(0-30)$ & $6.4(0-30)$ & $4.1(0-30)$ & 0.025 \\
\hline Días piso. (RI) & $13(0-62)$ & $10.9(0-45)$ & $11.4(0-60)$ & 0.730 \\
\hline \multicolumn{5}{|l|}{ Reintervenciones } \\
\hline LAPE (\%) & $5(22.7)$ & $8(21.6)$ & $6(10.71)$ & 0.262 \\
\hline $\mathrm{DBD}(\%)$ & $1(4.5)$ & $3(8.1)$ & - & 0.108 \\
\hline Retrasplante (\%) & $2(9)$ & $1(2.7)$ & - & 0.077 \\
\hline Muerte transoperatoria (\%) & $4(18.1)$ & $2(5.5)$ & - & 0.005 \\
\hline Muerte operatoria (\%) & $7(31.8)$ & $6(16.2)$ & $2(3.5)$ & 0.003 \\
\hline
\end{tabular}

DBD: derivación biliodigestiva; LAPE: laparotomía exploradora; NA: no aplica; PFC: plasma fresco congelado; PG: paquete globular; RI: rango intercuartílico;

SD: sin datos; TIF: tiempo de isquemia fría; TIT: tiempo de isquemia tibia; TQx: tiempo quirúrgico; UTI: unidad de terapia intensiva. 
Tabla 4. Complicaciones médicas tempranas divididas por etapas

\begin{tabular}{lcccc}
\hline & $\begin{array}{c}\text { Etapa 1 } \\
\mathbf{n = 2 2}\end{array}$ & $\begin{array}{c}\text { Etapa 2 } \\
\mathbf{n = 3 7}\end{array}$ & $\begin{array}{c}\text { Etapa 3 } \\
\mathbf{n = 5 6}\end{array}$ & $\mathbf{p}$ \\
\hline $\begin{array}{l}\text { Total } \\
\text { complicaciones }\end{array}$ & 47 & 24 & 29 & 0.001 \\
$\begin{array}{l}\text { Tempranas } \\
\text { Infecciones }\end{array}$ & 9 & 8 & 4 & 0.025 \\
Rechazo agudo & 9 & 8 & 8 & 0.537 \\
$\begin{array}{l}\text { Insuficiencia } \\
\text { renal aguda }\end{array}$ & 9 & 5 & 5 & 0.002 \\
$\begin{array}{l}\text { Complicaciones } \\
\text { pulmonares }\end{array}$ & 7 & 0 & 5 & 0.049 \\
Neurológicas & 2 & 1 & 5 & 0.407 \\
Otras & 11 & 2 & 2 & 0.001 \\
\hline
\end{tabular}

Tabla 5. Complicaciones médicas tardías divididas por etapas

\begin{tabular}{lcccc}
\hline & $\begin{array}{c}\text { Etapa 1 } \\
\mathbf{n = 2 2}\end{array}$ & $\begin{array}{c}\text { Etapa 2 } \\
\mathbf{n = 3 7}\end{array}$ & $\begin{array}{c}\text { Etapa 3 } \\
\mathbf{n}=\mathbf{5 6}\end{array}$ & $\mathbf{p}$ \\
\hline $\begin{array}{l}\text { Total } \\
\text { complicaciones }\end{array}$ & 7 & 21 & 9 & 0.001 \\
$\begin{array}{l}\text { Diabetes mellitus } \\
\text { Hipertensión }\end{array}$ & 0 & 4 & 2 & 0.945 \\
$\begin{array}{l}\text { arterial sistémica } \\
\text { Insuficiencia }\end{array}$ & 1 & 3 & 2 & 0.621 \\
$\begin{array}{l}\text { renal crónica } \\
\text { terminal }\end{array}$ & & 1 & 0 & 0.614 \\
Oncológicas & 0 & 3 & 0 & NA \\
Otras & 5 & 10 & 5 & 0.060 \\
\hline NA: no aplica. & & & &
\end{tabular}

\section{Discusión}

El presente estudio muestra los resultados del THO en el INCMNSZ, desde el primer THO en México ${ }^{15}$ hasta el año 2012. Decidimos dividir este periodo de tiempo en tres etapas. Consideramos la etapa 1 desde los inicios hasta 1999, cuando el procedimiento se realizaba de manera esporádica; etapa 2, del año 2000 hasta el año 2007, porque a pesar de que desde el año 2000 se contaba con un grupo multidisciplinario, en esta etapa nunca se realizaron 10 o más trasplantes por año; la etapa 3 fue, por lo tanto, de 2008 a 2012. Se tomó el año 2012 para que los receptores de trasplante tuvieran por lo menos un año de seguimiento. La evolución de nuestro programa, desde un centro pionero a nuestros días, ha mostrado una gran
Tabla 6. Complicaciones quirúrgicas divididas por etapas

\begin{tabular}{lcccc}
\hline & $\begin{array}{c}\text { Etapa 1 } \\
\mathbf{n = 2 2}\end{array}$ & $\begin{array}{c}\text { Etapa 2 } \\
\mathbf{n = 3 6}\end{array}$ & $\begin{array}{c}\text { Etapa 3 } \\
\mathbf{n = 5 6}\end{array}$ & $\mathbf{p}$ \\
\hline $\begin{array}{l}\text { Total } \\
\text { complicaciones }\end{array}$ & 11 & 18 & 15 & 0.002 \\
$\begin{array}{l}\text { Hemorragia del } \\
\text { sitio quirúrgico }\end{array}$ & 7 & 3 & 3 & 0.013 \\
$\begin{array}{l}\text { Complicaciones } \\
\text { biliares }\end{array}$ & 1 & 7 & 8 & 0.302 \\
$\begin{array}{l}\text { Complicaciones } \\
\text { vasculares }\end{array}$ & 0 & 2 & 4 & 0.539 \\
$\begin{array}{l}\text { Falla primaria del } \\
\text { injerto }\end{array}$ & 2 & 3 & 0 & 0.208 \\
Otras & 1 & 3 & 0 & 0.108 \\
\hline
\end{tabular}

Tabla 7. Complicaciones con la clasificación de Clavien-Dindo

\begin{tabular}{lcccc}
\hline $\begin{array}{l}\text { Clavien- } \\
\text { Dindo }\end{array}$ & $\begin{array}{c}\text { Etapa 1 } \\
\mathbf{n = 2 2}\end{array}$ & $\begin{array}{c}\text { Etapa 2 } \\
\mathbf{n = 3 7}\end{array}$ & $\begin{array}{c}\text { Etapa 3 } \\
\mathbf{n = 5 6}\end{array}$ & $\mathbf{p}$ \\
\hline I & 2 & $7(18.92)$ & $15(26.79)$ & 0.210 \\
II & - & $6(16.22)$ & $16(28.57)$ & 0.013 \\
III a & 2 & $6(16.22)$ & $12(21.43)$ & 0.422 \\
III b & 1 & $5(13.51)$ & $5(8.93)$ & 0.513 \\
IV a & 2 & $5(13.51)$ & $5(8.93)$ & 0.758 \\
IV b & 2 & - & $1(1.79)$ & 0.092 \\
V & 13 & $8(21.62)$ & $2(3.57)$ & 0.001 \\
\hline
\end{tabular}

diferencia en la última etapa (2008 a 2012), en la que se realizaron la mayoría de los THO $(56 / 117)$ y con los mejores resultados. Esta evolución del programa se ve manifiesta en el tipo de cirugía, pues en la etapa 1 predominó el bypass veno-venoso (77\%) y en las etapas 2 y 3 fue la exclusión total (95 y 98\%, respectivamente). Comparar las etapas 2 y 3 nos permite conocer de manera más clara la mejoría en cuanto a número y resultados del THO en nuestro centro, ya que se contaba exactamente con la misma infraestructura y desde el año 2000 se realiza el procedimiento de la misma forma. Con respecto al tiempo en lista de espera, este disminuyó de manera significativa de las etapas 1 y 2 a la etapa $3(365,657$ y 80 días, respectivamente; $p=0.0001$ ), gracias al incremento del número de donadores y a la asignación de órganos a través del MELD. Anteriormente, la asignación de órganos para trasplante hepático se realizaba por medio de la clasificación Child-Pugh, y se consideraban cuatro estadios de UNOS (United Network for Organ Sharing), 1A, 2A, 2B y 3, en los que el 


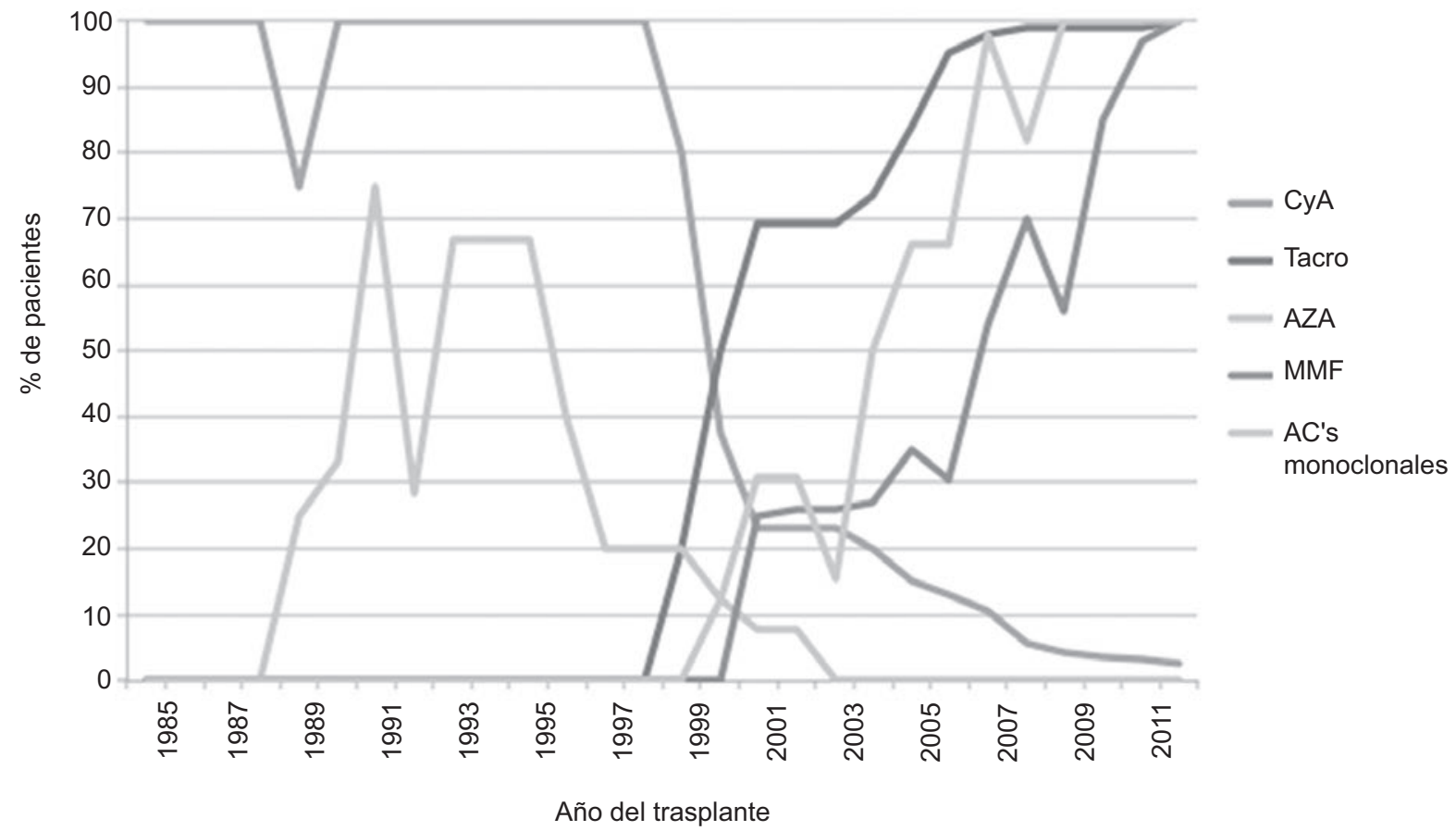

Figura 1. Inmunosupresión utilizada en el THO de los pacientes del INCMNSZ desde 1985 hasta 2012. Ac. monoclonales: anticuerpos monoclonales anti-CD25; AZA: azatioprina; CyA: ciclosporina; MMF: micofenolato de mofetilo; Tacro: tacrolimus.

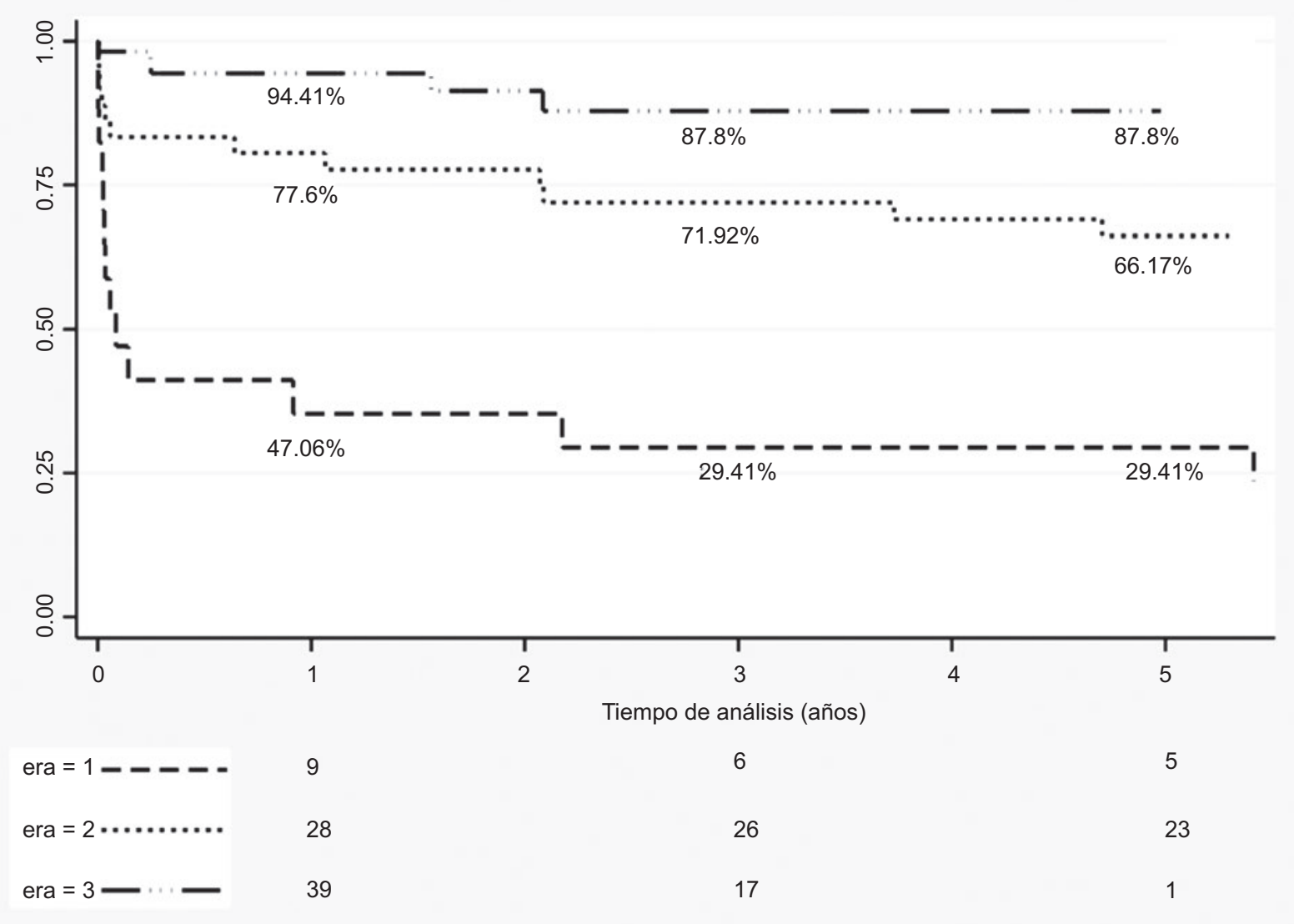

Figura 2. Supervivencia del THO dividida en etapas. 
tiempo en lista de espera era un factor muy importante para elegir al receptor. En el caso del MELD, se interviene a los pacientes más graves y el tiempo en lista de espera no es un factor determinante; por lo tanto, el tiempo en lista de espera es menor en la etapa del MELD. En nuestro centro, el MELD se empezó a utilizar en 2008 y por ello en la etapa 3 se realizó el trasplante a los pacientes más graves, aunque esto no mostró significancia estadística (etapa 214.5 vs. etapa $318.8 \% ; p=0.42$ ). Con respecto a las variables perioperatorias, observamos que tanto el tiempo quirúrgico como el uso de productos sanguíneos y la ocurrencia de sangrado fueron mayores en la etapa 3 que en la etapa 2. De primera instancia, no pareciera haber una mejoría en los resultados, pero cuando vemos variables de mayor importancia, como la mortalidad operatoria (3.5 vs. $21.7 \%$; $p=0.001)$ y la supervivencia a 1 y 5 años (94.4 y $87.8 \%$ vs. 77.6 y $66.7 \% ; p=0.001$ ), podemos apreciar la mejoría en los resultados (tabla 3). Las complicaciones médicas fueron muy similares entre las dos etapas y las complicaciones quirúrgicas disminuyeron de manera significativa de la etapa $2(48.6 \%)$ a la etapa $3(21.4 \%)$. En cuanto el reporte de complicaciones por su gravedad, con la clasificación de Clavien-Dindo se observa un mayor número de complicaciones de grado I y II en la etapa 3 que en la etapa 2; creemos que este mayor número se debe a que la información perioperatoria de los pacientes en la etapa 3 fue recolectada de manera prospectiva, y esto permite una mayor captación de complicaciones menores. Sin embargo, cuando observamos las complicaciones de grado III a $\mathrm{V}$, observamos de nuevo un menor número en la etapa 3 . Si tomamos en cuenta que, en el ámbito internacional, la mortalidad operatoria es del $5 \%$ y la supervivencia a 1 año es del $86 \%$, los resultados en nuestro Instituto en esta última etapa son mejores que los reportados internacionalmente, inclusive en centros de alto volumen, y son mucho mejores que los reportados de manera aislada por la mayoría de los centros nacionales ${ }^{16-20}$.

El THO es un procedimiento de alta complejidad que involucra múltiples factores críticos, tanto en el donador como en el receptor, y requiere un grupo multidisciplinario con experiencia. Aunque en otros tipos de cirugía de alta complejidad (cardiaca, pancreática, pulmonar, esofagectomías, traumatológica) se ha demostrado que un centro de alto volumen tiene mejores resultados que uno de bajo volumen ${ }^{21-23}$, en el caso del THO los resultados han sido inconcordantes. Los estudios iniciales de Edwards, et al. ${ }^{6} \mathrm{y}$ de Axelrod, et al. ${ }^{7}$ muestran que los pacientes en centros de alto volumen tienen menos mortalidad operatoria después del THO. Edwards, et al. ${ }^{6}$ analizaron todos los THO realizados en los EE.UU. de 1992 a 1994 (más de 7,000 pacientes) y observaron mejores resultados en los centros de alto volumen en la etapa anterior al MELD. Axelrod, et al. ${ }^{7}$, utilizando la base de datos del registro de trasplantes de los EE.UU., reportaron en el año 2004 un mayor riesgo, estadísticamente significativo, de muerte en los centros de bajo volumen de THO. Estudios posteriores de Northup, et al. ${ }^{11}$, Tracy, et al. ${ }^{24}$, y Reese, et al. ${ }^{25}$ examinaron estos mismos puntos después de la aparición del MELD y no encontraron relación directa entre el volumen y la supervivencia a 1 año. No pareciera que la diferencia en los resultados del THO reportados en estos estudios antes y después del MELD sea por falta de experiencia de los centros. Si bien los resultados del THO han mejorado con el paso del tiempo en todo el mundo, estos estudios analizan centros de bajo y alto volumen en el mismo periodo de tiempo. En los EE.UU y en Europa, para cada época, siempre ha habido una supervivencia a 1, 3 y 5 años mínima exigida para poder realizar este tipo de procedimiento.

En nuestro centro, en la etapa 3, aun realizando menos de 20 trasplantes por año, los resultados son excelentes tanto en mortalidad (3.5\%) como en supervivencia a 1 y 5 años (94.4 y 87.8\%, respectivamente). Además, en el año 2008 se inició la utilización del MELD y por lo tanto los receptores de trasplante en la etapa 3 se encontraban más graves que en la etapa 2 , aunque esta diferencia no fue estadísticamente significativa. A pesar de que en la etapa 2 se contaba también con un grupo multidisciplinario en THO, la mortalidad en ese periodo fue del $21.6 \%$, lo cual no es despreciable, e interesantemente nunca se llegó a 10 THO al año. Hasta donde conocemos, no existe ningún estudio que mencione cuál es el mínimo de trasplantes que deben realizarse por año en un centro. En nuestra experiencia, al comparar la etapa 2 con la etapa 3 parecería que realizar menos de 10 THO pudiera afectar los resultados. En la etapa 2, en 8 años no se observó mejoría a pesar de la experiencia global adquirida. En la etapa 3, el grupo quirúrgico cambió y por lo tanto no podemos pensar que los resultados mejoraron por una mayor experiencia global. Además del cambio en el grupo quirúrgico, el otro factor que consideramos como importante para los resultados es que a partir del año 2008 se han realizado más de 10 THO por año. Pensamos que esta información es valiosa, ya que en México, en el año 
Tabla 8. Características clínicas y demográficas de los donadores de la etapa 3

\begin{tabular}{|c|c|}
\hline Donadores: 55 & n/media (\%/mín-máx) \\
\hline Edad (años) & $27.7(10-64)$ \\
\hline Mujer & $21(38.2)$ \\
\hline Hombre & $34(61.8)$ \\
\hline Peso (kg) & $68(40-90)$ \\
\hline Talla (cm) & $165(136-185)$ \\
\hline IMC $\left(\mathrm{kg} / \mathrm{m}^{2}\right)$ & $24.6(18.4-35.6)$ \\
\hline \multicolumn{2}{|l|}{ Causa de muerte } \\
\hline TCE & $35(63.6)$ \\
\hline EVC & $14(25.5)$ \\
\hline Tumor del SNC & $4(7.3)$ \\
\hline Otras & $2(3.6)$ \\
\hline \multicolumn{2}{|l|}{ Uso de aminas } \\
\hline Sí & $51(92.7)$ \\
\hline No & $4(7.3)$ \\
\hline \multicolumn{2}{|l|}{ Biopsia } \\
\hline Normal & $36(65.5)$ \\
\hline Esteatosis grado I & $19(34.5)$ \\
\hline TAS $(\mathrm{mmHg})$ & $106(60-80)$ \\
\hline $\mathrm{TAD}(\mathrm{mmHg})$ & $62(30-110)$ \\
\hline $\mathrm{Hb}(\mathrm{g} / \mathrm{dl})$ & $11.7(6.4-18.7)$ \\
\hline Creatinina (mg/dl) & $1.02(0.3-2.6)$ \\
\hline $\mathrm{Na}^{+}(\mathrm{mEq} / \mathrm{dl})$ & $150.9(131-177)$ \\
\hline Bilirrubina total (mg/dl) & $0.74(0.1-3.1)$ \\
\hline Bilirrubina directa (mg/dl) & $0.28(0-2.4)$ \\
\hline AST (U/I) & $69.7(12-245)$ \\
\hline $\mathrm{ALT}(\mathrm{U} / \mathrm{I})$ & $48(10-210)$ \\
\hline Fosfatasa alcalina (U/l) & $76(24-257)$ \\
\hline GGT (U/l) & $29.1(8-145)$ \\
\hline INR & $1.3(0.8-3.2)$ \\
\hline \multicolumn{2}{|c|}{$\begin{array}{l}\text { ALT: alanina aminotransferasa; AST: aspartato aminotransferasa; } \\
\text { GGT: gamma glutamil transpeptidasa; Hb: hemoglobina; IMC: índice de masa corporal; } \\
\text { INR: International Normalized Ratio; EVC: enfermedad vascular cerebral; } \\
\text { SNC: sistema nervioso central; TAS: tensión arterial sistólica; TAD: tensión arterial } \\
\text { diastólica; TCE: traumatismo craneoencefálico. }\end{array}$} \\
\hline
\end{tabular}

2012, solo cuatro centros en todo el país realizaron 10 o más THO por año, a pesar de que hay 62 centros autorizados para realizarlos ${ }^{26}$. Desafortunadamente, en nuestro país, el reporte de resultados no es obligatorio y esto no permite tener un control adecuado de la calidad de cada centro, además de estar utilizando recursos renovables y no renovables (órganos) en grupos que no cuentan con un mínimo indispensable de supervivencia a 1, 3 y 5 años, así como una baja mortalidad perioperatoria. Los pocos reportes de resultados de centros mexicanos en revistas nacionales muestran que, en la mayoría de los casos, están muy por debajo de lo deseado por los estándares internacionales, y normalmente todos estos hospitales realizan menos de $10 \mathrm{THO}$ al año.

Esta es la primera vez que se demuestra, con un estudio de un centro mexicano de THO, que no es necesario ser un centro de mediano 0 alto volumen para obtener excelentes resultados, pero también sugiere que realizar menos de $10 \mathrm{THO}$ al año puede hacer que los resultados sean muy inferiores e inclusive inaceptables. De confirmarse esto, existiría una justificación lo suficientemente importante para cambiar la estrategia de las autoridades responsables en nuestro país de incrementar el número de centros para realizar más trasplantes, por centralizar este complejo procedimiento en grupos que realicen por lo menos 10 trasplantes al año.

Después de que un grupo adquiere la experiencia suficiente, probablemente realizar menos de 10 trasplantes por año no afecte los resultados en un inicio, pero eventualmente, si este procedimiento se vuelve muy esporádico, las posibilidades de errores en uno o varios pasos de esta compleja cirugía se incrementan. En nuestra experiencia, lo que ha ocurrido es que ha aumentado el número de trasplantes por año. En nuestro centro, este incremento es multifactorial: por un lado, se incrementó el número de cirujanos, anestesiólogos y hepatólogos, y por otro lado aumentó el número de ofertas de órganos.

En conclusión, el THO en nuestro Instituto es una realidad. A partir del año 2008 los resultados son excelentes, inclusive mejores que los de algunos centros de los EE.UU. y Europa, a pesar de no ser un centro de mediano o alto volumen. Pareciera que el número mínimo de THO es de 10 al año para poder lograr resultados similares a los reportados en el ámbito internacional.

\section{Agradecimientos}

Queremos hacer un reconocimiento especial a todos aquellos que en el pasado contribuyeron de manera importante al programa de THO del INCMNSZ: Dr. Héctor Orozco Zepeda ${ }^{\dagger}$, Dr. Héctor S. Diliz, Dr. Carlos Chan Núñez, Dr. Federico Chavez Peón', Dr. Víctor Acosta, Dr. Guillermo Castorena, Dr. Luis Guevara-González ${ }^{\dagger}$, Dr. David Kershenobich y Dr. Marco A. Olivera. Así como también a los directores del INCMNSZ, quienes apoyaron el programa desde su inicio: Dr. Manuel Campuzano, Dr. Donato 


\section{Alarcón ${ }^{\dagger}$, Dr. Fernando Gabilondo y Dr. David Kershenobich.}

\section{Bibliografía}

1. Vilatoba M, Eckhoff DE, Contreras JL. Selección del receptor para trasplante hepático. Rev Invest Clin. 2005;57:244-51.

2. Birkmeyer JD, Siewers AE, Finlayson EVA, et al. Hospital volume and surgical mortality in the United States. N Engl J Med. 2002;346:1128-37.

3. Halm EA, Lee C, Chassin MR. Is volume related to outcome in health care? A systematic review and methodologic critique of literature. Ann Intern Med. 2002;137:511-20.

4. Reames BN, Ghaferi AA, Birkmeyer JD, et al. Hospital volume and operative mortality in the modern era. Ann Surg. 2014;260:244-51.

5. Hosenpud JD, Breen TJ, Edwards EB, et al. The effect of transplant center volume on cardiac transplant outcome. A report of the United Network for Organ Sharing Scientific Registry JAMA. 1994;271:1844-9.

6. Edwards EB, Roberts JP, McBride MA, et al. The effect of the volume of procedures at transplantation centers on mortality after liver transplantation. N Engl J Med. 1999:341:2049-53.

7. Axelrod D, Guidinger M, McCulloug K, et al. Association of center volume with outcome after liver and kidney transplantation. Am J Transpl. 2004:4:920-7

8. Ahmad J, Bryce C, Cacciarelli T, et al. Differences in access to live transplantation: disease severity, waiting time, and transplantation cente volume. Ann Intern Med. 2007;146:707-13

9. Ozhathil D, Fu Li Y S Smith J, et al. Impact of center volume on outcomes of increased-risk liver transplants. Liver Transpl. 2011;17:1191-9.

10. Scarborough J, Pietrobon R, Tuttle-Newhall J, et al. Relationship between provider volume and outcomes for orthotopic liver transplantation. J Gastrointest Surg. 2008;12:1527-33.

11. Northup P, Pruett T, Stukenborg G, et al. Survival after liver transplantation does not correlate with transplant center case volume in the MELD era. Am J Transpl. 2006;6:2455-62.

12. Secretaría de Salud, Salud: México 2006 Información para la rendición de cuentas, 2007. (Septiembre 2017). http://www.salud.gob.mx/unidades/evaluacion/saludmex2006/SM06.pdf
13. Duro-García V, Niño-Murcia A. Report 2013. Latin America Transplantation. 2013:1:93

14. Feng S, Goodrich NP, Bragg-Gresham JL, et al. Characteristics associated with liver graft failure: the concept of a donor risk index. Am J Transpl. 2006;6:783-90.

15. Diliz H, Orozco H, Kershenobich D, et al. Liver transplantation in Mexico. Report of the first successful case. Rev Gastroenterol Mex. 1991;56:33-8.

16. Varela-Fascinetto G, Hernández-Plata JA, Nieto-Zermeño J, et al. Programa de trasplante hepático pediátrico en el Hospital Infantil de México Federico Gómez. Rev Invest Clin. 2011;63(Suppl 1):57-61

17. Hernández-Domínguez JM, Holm-Corzo A, Santos-Caballero M, et al. Experience in liver transplantation (1996-2011) at the UMAE, General Hospital Gaudencio Gonzalez Garza, National Medical Center La Raza, Mexican Institute of Social Security, Mexico City, D.F. Rev Invest Clin 2011;63(Suppl 1):62-6.

18. Cisneros-Garza LE, López-Hernández PA, Muñoz-Ramírez MR, et al. Trasplante hepático en la Unidad Médica de Alta Especialidad Num. 25 IMSS Monterrey. Rev Invest Clin. 2011;63(Suppl 1):69-72.

19. Rodríguez-Montalvo C, Tijerina-Gómez L, Flores-Villalba E, et al. Doce años de trasplante hepático continuo en el Hospital San José-Tec de Monterrey. Rev Invest Clin. 2011;63(Suppl 1):73-8.

20. Pérez-Rodríguez E, Muñoz-Espinosa LE, Zapata-Chavira $\mathrm{H}$, et al. Trasplante hepático ortotópico. Experiencia en el Hospital Universitario de Monterrey, N.L. Rev Invest Clin. 2011;63(Suppl 1):79-84

21. Bach PB, Cramer LD, Schrag D, et al. The influence of hospital volume on survival after resection for lung cancer. $\mathrm{N}$ Engl $\mathrm{J}$ Med. $2001 ; 345: 181-8$

22. Birmeyer JD, Siewers AE, Finalyson EV, et al. Hospital volume and surgical mortality in the United States. N Engl J Med. 2002;346:1128-37.

23. Rathore SS, Epstein AJ, Volpp KG, et al. Hospital coronary artery bypass graft surgery volume and patient mortality, 1998-2000. Ann Surg. 2004:239:110-7.

24. Tracy E, Bennett $\mathrm{K}$, Aviki $\mathrm{E}$, et al. Temporal trends in liver transplant centre volume in the USA. HPB. 2009;11:414-21.

25. Reese $\mathrm{P}$, Yeh $\mathrm{H}$, Thomasson $\mathrm{A}$, et al. Transplant center volume and outcomes after liver retransplantation. Am J Transpl. 2009;9:309-17.

26. CENATRA, Reporte Nacional 2012 de la Donación y Trasplantes, 2012. (Septiembre 2017). http://cenatra.salud.gob.mx/descargas/contenido/ trasplante/informe_anual_2014.pdf 